\title{
Long-Term survival (over 20 years), complete response and normal childhood development in medulloblastoma treated with antineoplastons AI 0 and AS2-I
}

\begin{abstract}
A 33-month-old male underwent a subtotal resection of his medulloblastoma of the cerebellum and brainstem in February 1994. Six weeks later, he began treatment at the Burzynski Clinic (BC) with intravenous antineoplastons A10 and AS2-1 (ANP) receiving average dose of $4.0 \mathrm{~g} / \mathrm{kg} / \mathrm{d}$ of A10 and $0.4 \mathrm{~g} / \mathrm{kg} / \mathrm{d}$ of AS2-1. The baseline magnetic resonance imaging (MRI) showed three lesions which resolved after six weeks of treatment. Follow-up MRIs confirmed complete response. Intravenous ANP was given for three years, followed with A10 and AS2-1 capsules $(0.12 \mathrm{~g} / \mathrm{kg} / \mathrm{d}$ each) for two years. The patient lives a normal life, over 20 years.
\end{abstract}

Keywords: Antineoplastons A10 and AS2-1, Clinical trials, Medulloblastoma, PNET, Signaling pathways
Volume 2 Issue 3 - 2015

\author{
Stanislaw S Burzynski, Gregory S Burzynski, \\ Ania Marszalek, Tomasz J Janicki, Juan F \\ Martinez-Canca \\ Burzynski Clinic, Houston, USA
}

\begin{abstract}
Correspondence: Stanislaw Burzynski, MD, PhD, Burzynski Clinic, 9432 Katy Freeway, Houston, TX 7755, USA, Tel 7I3-3355697, Fax 7|3-935-0649, Email srb@burzynskiclinic.com
\end{abstract}

Received: March 21, 2015 | Published: May 25, 2015
Abbreviations: BC, Burzynski Clinic; ANP, Antineoplastons A10 and AS2-1; MRI, Magnetic Resonance Imaging; MB, Medulloblastoma; RT, Radiation Therapy; CRR, Central Radiology Review; CR, Complete Response; WNT, Wingless Signaling Pathway; Hh, Hedgehog; MYC, oncogene of the ovarian Myelomatosis retrovirus; AKT, protein kinase B; RAS, Rat Sarcoma gene protein family; PN, Phenylacetate; PG, Phenylacetylglutaminate; PTEN, Phosphatase and Tensin Homolog; MAD, Myocyte Enhancer Factor 2D; CDK4, Cyclin-Dependent Kinase 4; CDK2, Cyclin-Dependent Kinase 2; PLK1, Polo-Like Kinase 1; CHK1, Checkpoint Kinase 1

\section{Introduction}

Medulloblastoma (MB) is the most common malignant brain tumor in the age group of 0-4 years. ${ }^{1}$ Patients with high-risk MB have postoperative residual tumor over $1.5 \mathrm{~cm} 2$ or metastasis. ${ }^{2}$ Standard treatment involves surgery, radiation therapy (RT) and chemotherapy. ${ }^{3}$ Such treatment improves the survival for patients with average-risk $\mathrm{MB}$, but the prognosis for high-risk MB remains poor. ${ }^{4}$ Late adverse effects include endocrine dysfunction, impaired growth and cognitive problems. ${ }^{4,5}$ Children below the age of three years are especially vulnerable and for them RT is seldom used. ${ }^{4}$ High-dose chemotherapy with autologous stem cell transplantation produce clinical benefits, but neuro-cognitive adverse effects continue to be devastating. ${ }^{6}$

Antineoplastons (ANP) A10 and AS2-1 are analogs of naturally occurring derivatives of glutamine, isoglutamine, and phenylacetic acid, which were submitted for a number of phase II clinical studies. ${ }^{7-11}$ This paper describes the case of a successful treatment and normal development of a patient less than 3 years of age with high-risk MB.

\section{Case presentation}

A 33-month-old male was diagnosed with $\mathrm{MB}$ in February 1994 with a 3 month history of headaches, nausea, vomiting, and a stumbling gate. The magnetic resonance imaging (MRI) of the head of February 25, 1994 revealed a large $(4 \mathrm{~cm} \mathrm{x} 4 \mathrm{~cm} \times 5 \mathrm{~cm})$ tumor involving the cerebellum and brainstem. Most of the tumor was contrast-enhancing. Initial therapy consisted of suboccipital craniectomy and subtotal removal of the tumor. The tumor had extended from the posterior fossa down to the upper aspect of the $\mathrm{C} 1$ ring, and up to the left inferior cerebellar peduncle. The majority of the tumor was removed. Postoperative MRI of the head of March 8, 1994 revealed hydrocephalus, postoperative changes and three nodules in the resection area, including one involving the cerebellum, measuring $0.8 \mathrm{~cm} \times 1.5 \mathrm{~cm}=1.2 \mathrm{~cm} 2,0.8 \mathrm{~cm} \times 1.0 \mathrm{~cm}=0.8 \mathrm{~cm} 2$ and $1.0 \mathrm{~cm} \times 1.5$ $\mathrm{cm}=1.5 \mathrm{~cm} 2$. The sum of the products of the two largest perpendicular diameters was $3.5 \mathrm{~cm} 2$ compared to preoperative $20 \mathrm{~cm} 2$. The size of the postoperative residual tumor over $1.5 \mathrm{~cm} 2$ classified this case as high-risk MB. On April 11, 1994, he was admitted for evaluation at the Burzynski Clinic (BC) in Houston, Texas. At that time, he was in good condition with his physical examination within normal limits. He started the treatment with ANP on April 13, 1994. A10 and AS2-1 were administered intravenously through subclavian vein catheter and double-channel infusion pump. The details of ANP administrations and evaluation were described previously., ${ }^{5,11-13}$ The patient was treated accordingly to Protocol CAN-01 under IND 43,742. He was treated with the average dose of $4.0 \mathrm{~g} / \mathrm{kg} / \mathrm{d}$ of A10, reduced to $2.9 \mathrm{~g} /$ $\mathrm{kg} / \mathrm{d}$ after 12 months, and $0.4 \mathrm{~g} / \mathrm{kg} / \mathrm{d}$ of AS2-1, divided into multiple infusions. The MRIs were evaluated by central radiology review (CRR). The follow-up MRI of the head of June 1, 1994 did not show any lesions indicating the beginning of complete response (CR). Three years of intravenous ANP was followed with A10 and AS21 capsules $(0.12 \mathrm{~g} / \mathrm{kg} / \mathrm{d}$ each) for two years. Additional prescription drugs included amoxicillin and allopurinol. Reversible toxicity included hypernatremia, grade 3 and anemia, grade 4. Follow-up MRI of June 1, 1994 did not show any tumor and additional MRIs, until October 2007, indicated CR confirmed by CRR (Figure 1). During the course of treatment and for an additional 20 years he was followed by physicians of $\mathrm{BC}$ together with his local pediatrician and neurologist. He developed normally, graduated from high school and enrolled in college. He received certification as a professional fireman. Recently he became happily married and is living a productive life.

\section{Discussion}

The described case accomplished rapid CR followed by normal childhood development. The options for successful treatment for him were limited. The data from profiling studies permitted to reach 
a consensus of at least four molecular subgroups of MB: group 1WNT, group 2- sonic hedgehog (Hh), group 3 and group $4 \cdot{ }^{14-16}$ At the time of this patient's diagnosis, molecular classification of MB was not available. The evaluation at the university hospital did not show evidence that it was the nodular desmoplastic type of MB, which has a good outcome without radiation and chemotherapy. It was the opinion of the consulting neuro-oncologist that he had a poor prognosis and that he will soon die without radiation and chemotherapy. On the other hand, he was not a candidate for radiation therapy due to his age. The main feature of high-risk MB is focal, high-level amplification of the $M Y C$ proto-oncogene. ${ }^{15-16}$ MYC promotes proliferation of MB cells and inhibits terminal differentiation. Several upstream signaling pathways including $A K T, R A S$, and apoptosis are implicated in tumorigenesis and potentiate activity of $M Y C$ (Figure 2). ${ }^{16}$ Apoptosis is promoted by the ingredients of ANP: phenylacetate (PN) and phenylacetylglutaminate $(\mathrm{PG})$
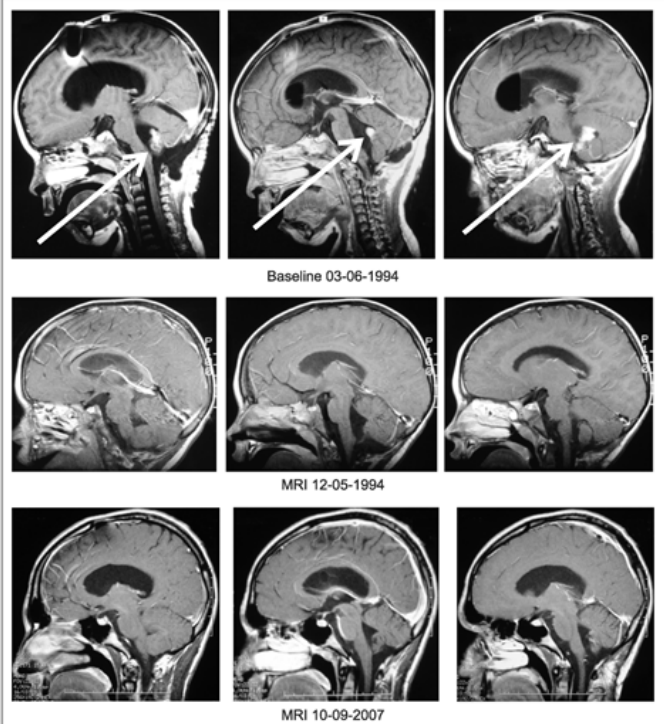

Fig. 1

Figure I Medulloblastoma in a 33-month-old male which recurred after subtotal resection. MRI of the head, TI enhanced baseline and two follow-up studies indicating complete response.

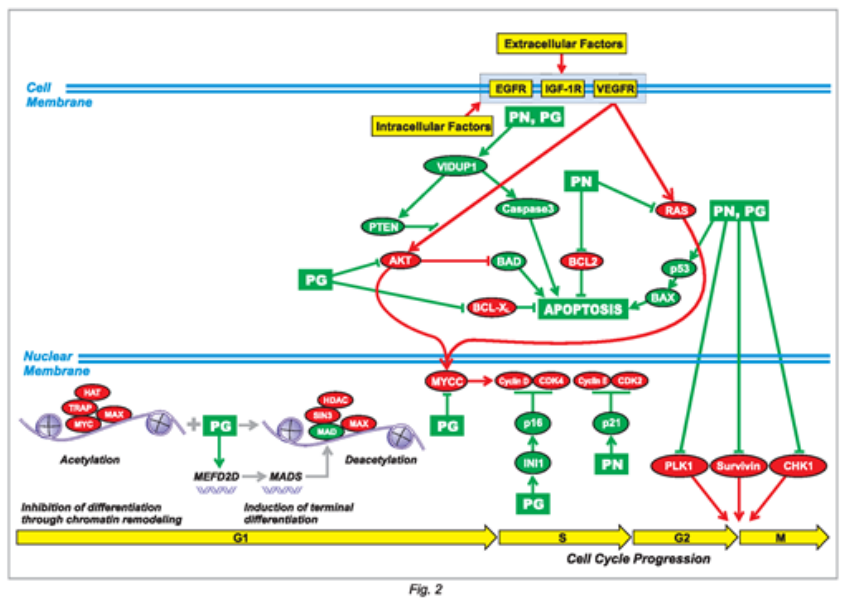

Figure 2 Proposed mechanism of action of antineoplastons AIO and AS2$I$ in group 3 medulloblastoma. The ingredients of antineoplastons AIO and AS2-I, PN and PG, effect signal transmission through AKT and RAS pathways, promote apoptosis, induce differentiation and interrupt cell cycle progression at GI/S and G2/M checkpoints. Abbreviations explained in the text.
The transduction of signal through $A K T$ and $R A S$ pathways are also interrupted by these active ingredients. ${ }^{12}$ The expression of the most important tumor suppressor genes PTEN and $p 53$ is increased by PN and $\mathrm{PG}^{8}{ }^{816}$ Inside the nucleus, $\mathrm{PG}$ is inducing terminal differentiation through replacement of MYC protein by MAD ${ }^{12}$ Induction of $M A D$ is facilitated by MADS box transcription enhancer factor (MEFD2D). PG decreases expression of $M Y C$ and interferes with the G1-S checkpoint through inhibition of cyclin D/cyclin-dependent kinase 4 (CDK4) complex. ${ }^{8,12} \mathrm{PN}$ increases expression of the $p 21$ tumor suppressor gene and inhibits cyclin E/CDK2 complex. ${ }^{8,12} \mathrm{PN}$ and PG decrease expression of polo-like kinase 1 (PLK1), survivin, and checkpoint kinase 1 (CHK1). ${ }^{17,18}$ This mechanism will need to be confirmed in additional studies.

\section{Acknowledgements}

The authors express their appreciation to the additional physicians involved in the care of the patients: Drs. Zanhua Yi, Alejandro Marquis, and Mohammad Khan. Preparation of the manuscript was provided by Carolyn Powers, Adam Golunski, and Jennifer Pineda.

\section{Conflicts of interest}

All authors are employed by Burzynski Clinic. Dr. Stanislaw $\mathrm{R}$ Burzynski is President at the Burzynski Clinic. Dr. Gregory S Burzynski is Vice-President of Burzynski Clinic and Dr. Sheldon Brookman is Director of Pharmaceutical Development of Burzynski Clinic.

\section{References}

1. Ostrom QT, Gittleman H, Liao P, et al. CBTRUS statistical report: primary brain and central nervous system tumors diagnosed in the United States 2007-2011. Neuro Oncol. 2014;16(Suppl 4):iv1-iv63.

2. Packer RJ, Rood BR, MacDonald TJ. Medulloblastoma: present concepts of stratification into risk groups. Pediatr Neurosurg. 2003;39(2):60-67.

3. NCCN Clinical Practice Guidelines in Oncology: Central Nervous System Cancers. National Comprehensive Cancer Network, Fort Washington, USA. 2012

4. PackerRJ, VezinaG. Managementofand prognosis withmedulloblastoma: therapy at a crossroads. Arch Neurol. 2008;65(11):1419-1424.

5. Burzynski SR. Treatments for astrocytic tumors in children: current and emerging strategies. Pediatr Drugs. 2006;8(3):167-178.

6. Sung KW, Lim DH, Son MH, et al. Reduced-dose craniospinal radiotherapy followed by tandem high-dose chemotherapy and autologous stem cell transplantation in patients with high-risk medulloblastoma. Neuro Oncol. 2013;15(3):352-359.

7. Burzynski SR. The present state of antineoplaston research (I). Integr Cancer Ther. 2004;3(1):47-58.

8. Burzynski SR, Janicki TJ, Burzynski GS, et al. The response and survival of children with recurrent diffuse intrinsic pontine glioma based on phase II study of antineoplastons A10 and AS2-1 in patients with brainstem glioma. Childs Nerv Syst. 2014;30(12):2051-2061.

9. Burzynski SR, Janicki TJ, Burzynski GS, et al. Long-term survival ( $>13$ years) in a child with recurrent diffuse pontine gliosarcoma: A case report. J Pediatr Hematol Oncol. 2014;36(7):e433-e439.

10. Burzynski SR, Janicki TJ, Burzynski GS, et al. A phase II study of antineoplastons A10 and AS2-1 in children with high-grade glioma. Final report (Protocol BT-06), and review of recent trials. $J$ Cancer Ther. 2014;5(6):565-577.

11. Burzynski SR, Janicki T, Burzynski G, A phase II study of antineoplastons A10 and AS2-1 in children with recurrent, refractory or progressive primary brain tumors - Final report (Protocol BT-22). J Cancer Ther. 2014;5(10):977-988. 
12. Burzynski SR, Weaver RA, Janicki T, et al. Long-term survival of high-risk pediatric patients with primitive neuroectodermal tumors treated with antineoplastons A10 and AS2-1. Integr Cancer Ther. 2005;4(2):168-177.

13. Burzynski SR, Conde AB, Peters A, et al. A retrospective study of antineoplastons A10 and AS2-1 in primary brain tumours. Clin Drug Invest. 1999;18(1):1-10.

14. Taylor MD, Northcott PA, Korshunov A, et al. Molecular subgroups of medulloblastoma: the current consensus. Acta Neuropathol. 2012;123(4):465-472.

15. Northcott PA, Jones DTW, Kool M, et al. Medulloblastomics: the end of the beginning. Nature Rev Cancer. 2012;12(12):818-834.
16. MacDonald TJ, Aguilera D, Castellino RC. The rationale for targeted therapies in medulloblastoma. Neuro Oncol. 2014;16(1):9-20.

17. Patil SS, Burzynski SR, Mrowczynski E, et al. Phenylacetylglutaminate and phenylacetate in combination upregulate VDUP1, cause cell cycle blockade and apoptosis in U87 glioblastoma cells. $J$ Cancer Ther. 2012;3(3):192-200.

18. Triscott J, Lee C, Foster C, et al. Personalizing the treatment of pediatric medulloblastoma: Polo-like kinase 1 as a molecular target in high-risk children. Cancer Res. 2013;73(22):6734-6744. 